\title{
Teleasistencia psicológica para niños en edad escolar con Trastorno por Déficit de Atención/Hiperactividad: Rol de los padres de familia y cuidadores
}

\author{
Connie Aliaga Guanilo ${ }^{12}$, Caroline Millones Choquicondo ${ }^{12}$, \\ Astry Torres-Chamorro ${ }^{12}$ y Betsabe Vasquez-Florentino ${ }^{12}$ \\ ${ }^{1}$ Instituto Psicopedagógico EOS Perú \\ ${ }^{2}$ Universidad Nacional Mayor de San Marcos
}

\section{Resumen}

Ante el contexto de pandemia por COVID-19, se han tomado diversas medidas de control, siendo una de ellas la cuarentena, originando así, una serie de consecuencias en la sociedad, como la imposibilidad de asistir a terapias presenciales. Esto último, ha generado que muchos niños con algún tipo de condición como, por ejemplo, niños con Trastorno por Déficit de Atención/Hiperactividad (TDAH), se vuelvan vulnerables, provocando que se acentúen los síntomas del trastorno. Por lo que, algunos padres y profesionales, han optado por el uso de la teleasistencia psicológica como alternativa de trabajo, con el fin de continuar con las terapias. Por tal motivo, el presente trabajo tiene como objetivos: (1) informar sobre la viabilidad y aceptabilidad de la teleasistencia psicológica, (2) describir el rol de los padres de familia y cuidadores en la teleasistencia psicológica, y (3) brindar una guía para padres y cuidadores de niños con TDAH para el manejo en casa durante el contexto de cuarentena.

Palabras clave: entrenamiento para padres, intervenciones basadas en evidencia, recursos tecnológicos, teleasistencia psicológica, TDAH.

\section{Introducción}

\section{Trastorno por Déficit de Atención/ Hiperactividad (TDAH)}

El Trastorno por Déficit de Atención/ hiperactividad (TDAH) es un trastorno de conducta infantil común caracterizado por inatención y/o hiperactividad e impulsividad que comienza en el período del desarrollo (American Psychiatric Association, 2013).

La inatención se visualiza conductualmente como desviaciones en las tareas, falta de persistencia, dificultad para mantener la atención y desorganización, que no se deben a un desafío o falta de comprensión. Mientras que, la hiperactividad-impulsividad se manifiesta como conductas motoras excesivas y apresuradas que se producen en el momento, sin reflexión, y que generan un gran riesgo de daño al individuo (American Psychiatric Association, 2013; Organización Mundial de la Salud; Sayal et al., 2017).

EI TDAH es más común en niños que en niñas, con una proporción de 02-03 a 01 y a nivel mundial afecta al 5\% de los niños en edad escolar (Holland \& Sayal, 2018; Sayal, 2017).
Además, se ha encontrado que de este trastorno se deslinda una amplia variedad de problemas, que incluyen comportamientos desafiantes, disruptivos y antisociales, problemas emocionales y bajo rendimiento en la escuela (Sayal et al., 2017).

Viabilidad y aceptabilidad de la Teleasistencia

La teleasistencia es una alternativa de trabajo viable para proporcionar evaluaciones e intervenciones basadas en evidencia (Aliaga et al., 2020; Spencer et al., 2019). No solo existen pruebas sólidas de su efectividad y viabilidad, sino también alto agrado por parte de los padres de niños con TDAH, que consideran aceptable el uso de tecnologías no presenciales, calificándolas como muy satisfactorias (Rismo et al., 2016; Owen, 2019).

El presente trabajo tiene como objetivos: (1) informar sobre la viabilidad y aceptabilidad de la teleasistencia psicológica, (2) describir el rol de los padres de familia y cuidadores en la teleasistencia psicológica, y (3) brindar una guía para padres y cuidadores de niños con TDAH para el manejo en casa durante el contexto de cuarentena. 


\section{Consideraciones para la teleasistencia psicológica basada en evidencia científica}

\section{Público objetivo}

Dirigido a psicólogos, especialistas, internistas, padres de familia y cuidadores de niños, de 06 a 12 años, con Trastorno por Déficit de Atención/Hiperactividad (TDAH).

\section{Estrategias y técnicas}

Para garantizar una atención psicológica de calidad, es necesario que los psicólogos, especialistas y otros miembros involucrados (por ejemplo: los internistas) estén altamente calificados en el uso de estrategias y técnicas basadas en evidencia científica, pues así podrán realizar una adecuada evaluación e intervención psicológica (Fischer et al., 2017; Sandbank et al., 2020). Es decir, no solo deben conocer las estrategias y técnicas existentes, sino también saber aplicarlas y decidir cuáles son las más adecuadas y oportunas para cada caso que se les presente. Por ello, a continuación, se mencionan aquellas estrategias y técnicas que han resultado eficaces en el sistema de teleasistencia para niños con TDAH (Tabla 1).

Diversos estudios indican que las intervenciones conductuales, basadas en los principios del condicionamiento operante y la teoría del aprendizaje social, son las más eficaces en el tratamiento de niños con TDAH (Chronis-Tuscano et al., 2016; Evans et al., 2014; Juhn et al., 2015; Schechter \& Kollins, 2019). Así mismo, estos estudios han identificado al control de antecedentes, atención diferencial, modelado, reforzamiento, observación conductual y al análisis funcional de la conducta como las estrategias y técnicas más relevantes para la evaluación e intervención de forma remota en niños con TDAH.

Rol de los padres de familia y cuidadores en la teleasistencia psicológica

Se ha encontrado que la participación de los padres es fundamental durante todo el proceso de evaluación e intervención, puesto que, solo así se podrá lograr la generalización del tratamiento a través del tiempo y en distintos contextos (Chronis-Tuscano et al., 2016; Spencer et al., 2019). De esta forma, los padres y cuidadores ocupan un rol importante dentro del sistema de teleasistencia, en este caso, para niños con TDAH (DuPaul et al., 2017; Schechter \& Kollins, 2019).

Por otro lado, la participación de los padres y cuidadores en la teleasistencia psicológica (Tabla 1) irá cambiando a partir de dos variables: (1) la edad que tenga el niño y (2) su sintomatología (Juhn et al. 2017; Myers et al., 2015; Schechter \& Kollins, 2019; VanderStoep et al., 2017).

En caso la intervención esté dirigida a niños que tienen entre 06 y 08 años, es necesario que los padres y cuidadores tomen un rol totalmente activo, ya que, serán ellos quienes apliquen las estrategias y técnicas, que deberán ser proporcionadas por el especialista través de un programa de asesoramiento y entrenamiento (DuPaul et al., 2017; Rismo et al., 2016).

Por otra parte, si la intervención está dirigida a niños que tienen entre 09 y 12 años, los padres y cuidadores cumplirán un rol de monitoreo o supervisión, debido a que, si bien ellos continuarán recibiendo asesoramiento y entrenamiento, el niño también podrá participar de forma directa en la teleasistencia por medio de algunas sesiones programadas con el especialista; que deberán estar siempre bajo la supervisión de los padres con el fin de tener controlado el ambiente y evitar posibles distracciones por parte del menor durante la intervención (Myers et al., 2015; Sayal et al., 2017; Rismo et al., 2016). No obstante, si el nivel de sintomatología manifestado en el niño dificulta su participación en las sesiones de modalidad online, la intervención deberá ser igual que del grupo de 06 a 08 años (Myers et al., 2015; Sayal et al., 2017).

Cabe resaltar que, esta división de edades (de 06 a 08 años y de 09 a 12 años) se realiza a partir de la revisión de diversos estudios. Estos mencionan que mientras más pequeño sea el niño o mayor sintomatología presente, es mejor que las intervenciones de modalidad online sean directamente con los padres (Juhn et al., 2015; Myers et al., 2015; Rismo et al., 2016; Sayal et al., 2017; Schechter \& Kollins, 2019).

\section{Instrumentos y recursos tecnológicos}

Con respecto al uso de instrumentos para la evaluación de forma remota, es relevante mencionar que no todos los instrumentos podrán ser adaptados a una modalidad online 
y algunos que ya se encuentran en esa modalidad pueden tener costos elevados; por lo que, se recomienda optar, primordialmente, por el uso de listas de cotejo, la entrevista psicológica y el análisis funcional de la conducta para la realización de una buena evaluación (Aliaga et al., 2020).

En relación con los recursos tecnológicos, se recomienda el uso de las computadoras o laptops como mejores recursos tangibles; así mismo, se recomienda el uso de plataformas (recursos intangibles) como Microsoft Teams, Line y Google Hangouts para las intervenciones por medio de videoconferencias (Aliaga et al., 2020). Todo ello con el fin de asegurar la confidencialidad y privacidad de los datos durante la teleasistencia psicológica (Colegio Logopedas de Murcia, 2020)

Tabla 1

Estrategias, técnicas y rol de los participantes en la teleasistencia psicológica

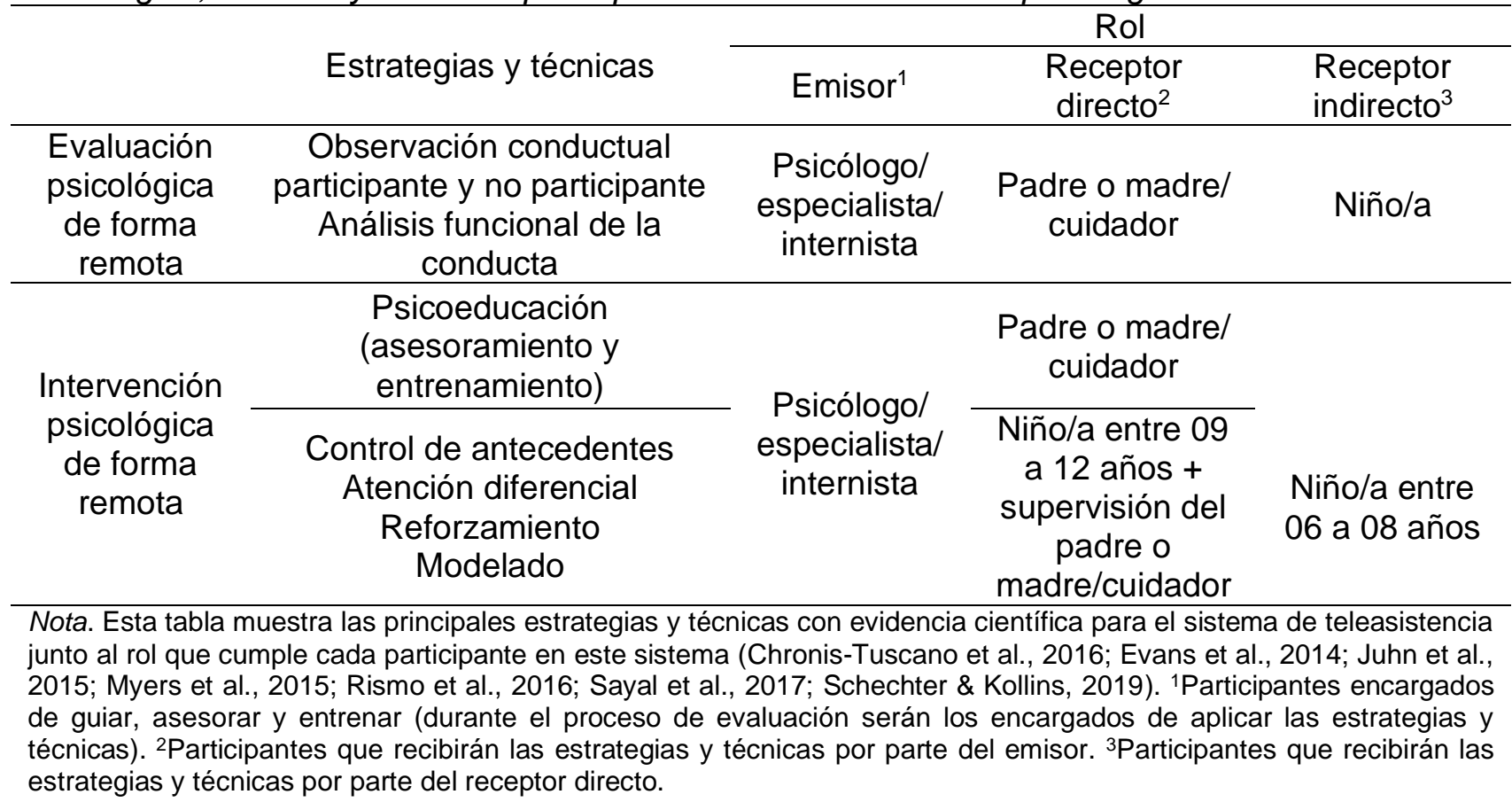

\section{Guía para padres y cuidadores de niños con TDAH: Manejo en casa durante el contexto de cuarentena}

El aislamiento social o contexto de cuarentena podría colocar en una posición, particularmente, vulnerable a un niño con TDAH, provocando que se acentúen los síntomas del trastorno (Cortese et al., 2020). Así mismo, los padres podrían presentar altos niveles de estrés, puesto que, al no contar con asesorías profesionales o espacios donde el niño pueda ser atendido, se presentan mayores dificultades en el control y manejo de conductas problemáticas (Toseeb, et al., 2020).

Por ello, el presente trabajo brinda una guía para padres y cuidadores (Barkley, 2013;
Cortese et al., 2020) con el fin de dar a conocer una serie de pautas para el manejo conductual de niños con TDAH durante esta situación de aislamiento social (Anexo 1).

\section{Conclusiones}

La teleasistencia psicológica ha recibido mayor aceptabilidad por parte de los padres de niños con TDAH, considerándola como una alternativa de trabajo muy satisfactoria. Por lo que, es importante seguir conociendo estrategias y técnicas que se puedan adaptar a esta modalidad con el fin de garantizar en el paciente una atención de calidad.

Futuras investigaciones deberán abordar las barreras, facilidades y fiabilidad de la implementación de la teleasistencia 
psicológica en adolescentes y adultos con TDAH.

\section{Referencias}

Aliaga, C., Lopez-Fernández, T., TorresChamorro, A., y Vasquez-Florentino, B. (2020). Teleasistencia psicológica para niños con Trastorno del Espectro Autista: definiciones, directrices $y$ tendencias tecnológicas para el trabajo basado en evidencia. https://doi.org/10.31219/osf.io/2auvn

American Psychiatric Association (2013). Manual diagnóstico y estadístico de los trastornos mentales (DSM-5). Médica Panamericana.

Barkley, R. A. (2013). Taking charge of ADHD: The complete, authoritative guide for parents (3ra ed.). Guilford press.

Chronis-Tuscano, A., Wang, C., Woods, K., Strickland, J., \& Stein, M. (2016). Parent ADHD and Evidence-Based Treatment for Their Children: Review and Directions for Future Research. Journal of Abnormal Child Psychology, 45(3), 501-517. https://doi.org/10.1007/s10802-0160238-5

Colegio Logopedas de Murcia (2020). Guía Teleasistencia Logopedia COVID-19. Consejo General de Colegios de Logopedas. https://cutt.ly/lyaaTE4

Cortese, S., Asherson, P., Sonuga-Barke, E., Banaschewski, T., Brandeis, D., Buitelaar, J., Coghill, D., Daley, D., Danckaerts, M., Ditmann, R., Doepfner, M., Ferrin, M., Hollis, C., Holtmann, M., Konofal, E., Lecendreux, M., Santosh, P., Rothenberger, A., Soutullo, C., Steinhausen, H.-C., ... Simonoff, E. (2020). ADHD management during the COVID-19 pandemic: guidance from the European ADHD Guidelines Group. The Lancet Child \& Adolescent Health. https://doi.org/10.1016/S23524642(20)30110-3

DuPaul, G., Kern, L., Belk, G., Custer, B., Hatfield, A., Daffner, M., \& Peek, D. (2017). Promoting Parent Engagement in Behavioral Intervention for Young Children With ADHD: Iterative Treatment Development. Topics in
Early Chilhood Special Education, 38(1).

https://doi.org/10.1177/027112141774 6220

Evans, S. W., Owens, J. S., \& Bunford, N. (2014). Evidence-based psychosocial treatments for children and adolescents with attention-deficit/hyperactivity disorder. Journal of Clinical Child and Adolescent Psychology, 43, 527-551. https://doi.org/10.1080/15374416.2017 .1390757

Fischer, A. J., Clark, R., Askings, D., \& Lehman, E. (2017). Technology and telehealth applications. In J. K. Luiselli (Ed.), Applied behavior analysis advanced guidebook: $A$ manual for professional practice (p. 135-163). Elsevier Academic Press. https://doi.org/10.1016/B978-0-12811122-2.00007-3

Holland, J. \& Sayal, K. (2018). Relative age and ADHD symptoms, diagnosis and medication: a systematic review. European Child \& Adolescent Psychiatry, 60(4). https://doi.org/10.1007/s00787-0181229-6

Juhn, Y., McCarty, C., Vander-Stoep, A., \& Myers, K. (2015). Teletherapy Delivery of Caregiver Behavior Training for Children with Attention-Deficit Hyperactivity Disorder. Telemedicine Journal and e-health, 21(6), 451-458. https://doi.org/10.1089/tmj.2014.0132

Myers, K., Vander-Stoep, A., Zhou, C., McCarty, C., \& Katon, W. (2015). Effectiveness of a Telehealth Service Delivery Model for Treating AttentionDeficit/Hyperactivity Disorder: A Community-Based Randomized Controlled Trial. Journal of the American Academy of Child \& Adolescent Psychiatry, 54(4), 263-274. https://doi.org/10.1016/j.jaac.2015.01. 009

Organización Mundial de la Salud (2019). Clasificación Internacional de Enfermedades (CIE-11). http://cutt.ly/jysquR9 
Owen, N. (2019). Feasibility and acceptability of using telehealth for early intervention parent counselling. Advances in Mental Health, 18(1), 39-39. https://doi.org/10.1080/18387357.2019 .1679026

Rismo, M., Casado-Lumbreras, C., \& ColomoPalacios, R. (2016). ADHD in eHealth a systematic literature review. Procedia Computer Science, 100, 207-214. https://doi.org/10.1016/j.procs.2016.09 .142

Sandbank, M., Bottema-Beutel, K., Crowley, S., Cassidy, M., Dunham, K., Feldman, J. I., Crank, J., Albarran, S. A., Raj, S., Mahbub, P., \& Woynaroski, T. G. (2020). Project AIM: Autism intervention meta-analysis for studies of young children. Psychological Bulletin, 146(1), 129. https://doi.org/10.1037/bul0000215

Sayal, K., Prasad, V., Daley, D., Ford, T., \& Coghill, D. (2017). ADHD in children and young people: prevalence, care pathways, and service provision. The Lancet Psychiatry, 5(2), 175-186. https://doi.org/10.1016/S22150366(17)30167-0

Schechter, C., \& Kollins, S. (2019). Adaptations to Behavioral Parent Training for Children with ADHD: Promoting Access, Engagement, and Treatment Effectiveness. The ADHD Report, 27(1). https://cutt.ly/pyvJKor

Spencer, T., Noyes, E., \& Biederman, J. (2019). Telemedicine in the management of ADHD: Literature Review of Telemedicine in ADHD. Journal of Attention Disorders, 24(1). https://doi.org/10.1177/108705471985 9081

Toseeb, U., Asbury, K., Code, A., Fox, L., \& Deniz, E. (2020). Supporting families with children with Special Educational Needs and Disabilities during COVID19. PsyArXiv. https://doi.org/10.31234/osf.io/tm69k

Vander-Stoep, A., McCarty, C., Zhou, C., Rockhill, C., Schoenfelder, E., \& Myers, K. (2017). The Children's AttentionDeficit Hyperactivity Disorder
Telemental Health Treatment Study: Caregiver Outcomes. Journal of Abnormal Child Psychology, 45, 27-43. https://doi.org/10.1007/s10802-0160155-7 


\section{Anexos}

Anexo 1. Guía para padres y cuidadores de niños con TDAH: Manejo en casa durante el contexto de cuarentena.

\section{Atención}

1. Dé instrucciones claras

Asegúrese que su hijo le esté prestando atención. No presente la instrucción como pidiendo un favor y no dé más de una indicación a la vez. Puede pedirle al niño que repita la orden para confirmar que la ha entendido.

2. Cree rutinas

Haga una rutina para su hijo y síganla todos los días, considerando la actividad física dentro de los hábitos. Establezca rituales alrededor de las comidas, la tarea, la hora de jugar y la hora de dormir, con tareas diarias simples como seleccionar la ropa del día siguiente.

3. Establezca un lugar específico para tareas Elija un lugar donde su hijo pueda hacer su tarea en casa. Asegúrese de que esté alejado de distracciones como la televisión, otras personas $u$ objetos como una ventana.

4. Divida el tiempo de estudio

Divida las tareas para que no tenga que hacerlo todo de una vez. En lugar de un bloque largo único, haga que aborde tres sesiones de 20 minutos con un descanso entre ellas o cambiando el tema de la tarea. Al igual que las tareas diarias, divida los grandes proyectos a largo plazo en pasos simples. Establezca un horario con una fecha de vencimiento para cada paso. Estos pequeños plazos lo ayudarán a completar el proyecto a tiempo.

5. Asegúrese de entregar la tarea.

Es posible que su hijo pase horas haciendo su tarea, luego la pierda o se olvide de entregarla. Un sistema organizado de carpetas con subcarpetas para nuevas tareas y tareas terminadas, puede ayudarlo a entregar los trabajos con los límites de tiempo.

\section{Hiperactividad/impulsividad}

1. Motive con recompensas

Felicite a su hijo cuando hace un buen trabajo. También puede utilizar un sistema de puntos, permitiéndole acumularlos para después canjear una recompensa como ver televisión o jugar videojuegos.

2. Defina las reglas, pero permita cierta flexibilidad

Primero decida qué comportamientos tolerará y no tolerará. Consentir un comportamiento un día y no aceptarlo al siguiente es perjudicial para la mejora de un niño. Algunas conductas deben ser inaceptables, como los arrebatos físicos. Sin embargo, recuerde que los niños con TDAH pueden no adaptarse al cambio tan bien como otros. Permita que su hijo cometa errores a medida que aprende.

3. Regule los patrones de sueño

La falta de sueño exacerba la falta de atención, la hiperactividad y la imprudencia. Para ayudarlos a descansar mejor, elimine los estimulantes como el azúcar y la cafeína, y disminuya el tiempo de televisión. Establezca un ritual saludable y relajante antes de acostarse.

4. Fomente el pensamiento en voz alta

Los niños con TDAH pueden carecer de autocontrol. Esto hace que hablen y actúen antes de pensar. Pídale a su hijo que verbalice sus pensamientos $y$ razonamientos cuando surja la necesidad de actuar.

5. Promueva el tiempo de espera

Otra forma de controlar el impulso de hablar antes de pensar es enseñarle a su hijo a hacer una pausa antes de hablar 0 responder. Esto puede ayudar también a manejar las agresiones. 
6. Fomente el ejercicio

La actividad física tiene un efecto favorable en el proceso de aprendizaje. Puede ayudar al niño con TDAH a encontrar una manera constructiva de enfocar su pasión, atención y energía, disminuyendo su impulsividad y enfocándose en los movimientos del cuerpo. Destine un lugar en la casa donde pueda realizar actividad física (a través de juegos) con su hijo.

\section{Estrés parental}

1. Encuentre asesoramiento online individualizado

Su hijo necesita su aliento, pero Ud. también necesita ayuda profesional. No tenga miedo de buscar ayuda si la necesita, ya que un terapeuta puede ayudarlo a controlar su estrés y ansiedad, así como a su hijo.

2. Tome descansos

Es normal sentirse abrumado o frustrado consigo mismo o con su hijo. Así como su hijo necesitará tomar descansos mientras estudia, Ud. también puede tomar sus propios descansos. Programar tiempo a solas es importante para cualquier padre.

3. Utilice el tiempo de espera

Los niños imitan los comportamientos que ven a su alrededor, por lo que, si permanece controlado durante un estallido, ayudará a su hijo a hacer lo mismo. Tómese el tiempo para respirar, relajarse y ordenar sus pensamientos antes de intentar calmar a su hijo.

4. Está bien sentir estrés

Es normal sentirse impotente y que la relación con su hijo se vuelva hostil, negativa 0 estresante, sin embargo, recuerda que cada paso cuenta, y que tiene la libertad de elegir las acciones que hace y cómo reacciona luego de estas situaciones, tómese un tiempo para relajarse. 